\title{
Unification of Fermion and Boson Stochastic Calculus *
}

\author{
R. L. Hudson ${ }^{1}$ and K. R. Parthasarathy ${ }^{2}$ \\ ${ }^{1}$ Mathematics Department, University of Nottingham, University Park, \\ Nottingham NG7 2RD, England \\ 2 Indian Statistical Institute, 7 SJS Sansanwal Marg, New Delhi 110016, India
}

\begin{abstract}
Fermion annihilation and creation processes are explicitly realised in Boson Fock space as functions of the corresponding Boson processes and second quantisations of reflections. Conversely, Boson annihilation and creation processes can be constructed from the Fermion processes. The existence of unitary stochastic evolutions driven by Fermion and gauge noise is thereby reduced to an equivalent Boson problem, which is then solved.
\end{abstract}

\section{Introduction}

In [3] we constructed, out of the Boson field commutation relations, a quantum stochastic calculus including an Ito product formula. Existence of solutions of a corresponding class of quantum stochastic differential equations was proved, leading to unitary operator valued processes which, together with their generalisations [4], provide a natural method of dilating quantum dynamical semigroups. In its simplest form the Boson stochastic calculus uses as integrators the Boson annihilation and creation operators corresponding to the indicator function $\chi_{[0, t]}$ in $L^{2}\left(\mathbb{R}_{+}\right)$, together with the differential second quantisation of the operator of multiplication by $\chi_{[0, t]}$, the latter being called the gauge process.

In [1] a partial analog of this theory was given, in which Fermion second quantisation replaces Boson. The Fermion theory contains that of the Ito-Clifford integral [2] just as the Boson contains that of classical Brownian motion [3]. However no Fermion analog of the gauge process was used, and the unitary processes constructed were artificially restricted to being even with respect to the natural $\mathbb{Z}_{2}$-grading of Fermion Fock space and an assumed $\mathbb{Z}_{2}$-grading of the initial space. While this work was being prepared we received a preprint [8] which remedies these deficiencies.

* This work was carried out while both authors were participating in the Symposium on Stochastic Differential Equations at the University of Warwick. The first author acknowledges conversations with R.F. Streater during the same Symposium 
The primary purpose of our present work is to show that Fermion annihilation and creation processes can be realised in Boson Fock space by means of a simple stochastic integral prescription [see (3.5)], in such a way that the filtrations generated by the Boson annihilation and creation processes, and by the corresponding Fermion processes, coincide. In particular a natural identification is obtained between Boson and Fermion Fock spaces over $L^{2}\left(\mathbb{R}_{+}\right)$. The gauge process for Fermions is seen to be identical to that for Bosons. In this connection we note the recent preprint [6], in which operators $b_{k}, k=1, \ldots, n$ are constructed in Fermion Fock space for finitely many degrees of freedom which satisfy an approximate form of Boson commutation relations. The formula

$$
(-1)^{n} f_{k}=(-1)^{k} b_{k}
$$

relating the Fermion operators $f_{k}$ to these approximate Boson operators may be regarded as a discrete form of our Eq. (3.5). P. A. Meyer informs us that he has a realisation of Fermion processes in $L^{2}(w)$, where $w$ is Wiener measure [7].

Since completing this work we learned of the works [9] in which Fermion fields are constructed in Boson Fock space. The stochastic calculus gives a simple method of effecting such constructions [10]. We are grateful to R. F. Streater for drawing our attention to this work, and to P. Garbaczewski for correspondence and the preprint [10].

The construction of Fermionic unitary processes may now be undertaken profitably from this point of view. In particular the defining stochastic differential equation reduces to a corresponding Boson equation, for which techniques based on coherent states are available avoiding the lengthy inductive methods based on finite-particle Fermion states of [1] (and [8]). The Boson equation requires an extension of the method of [3], in so far as an operator process which does not lie entirely in the initial space enters into the driving coefficients (as in [5]). The proof of unitarity is based on reduction to a system of four coupled ordinary differential equations, instead of the single equation of [3]. The resulting Fermion unitary processes are no longer restricted to be even, and incorporate the gauge process as a driving noise term.

We use the following notational conventions. Hilbert space inner products, denoted by $\langle$,$\rangle are linear on the right. Operators T$ and $T^{\dagger}$ are said to be mutually adjoint if $\left\langle T^{\dagger} \phi_{1}, \phi_{2}\right\rangle=\left\langle\phi_{1}, T \phi_{2}\right\rangle$ for arbitrary $\phi_{1}$ in the domain of $T^{\dagger}$ and $\phi_{2}$ in the domain of $T . S^{c}$ denotes the complement of a set $S . \int_{a}^{b} f$ means the Lebesgue integral of $f$ over $[a, b] . F_{1} \ldots \hat{F}_{j} \ldots F_{n}$ means $F_{1} \ldots F_{j-1} F_{j+1} \ldots F_{n}$. A statement involving the symbol \# represents the two statements obtained respectively by deleting it and replacing it by the symbol $\uparrow$. We denote by $\varsigma_{n}$ the group of permutations of $\{1, \ldots, n\}$, and by $\sigma(\pi)$ the sign of an element $\pi \in \sigma_{n}$. We denote by per $C$ the permanent $\sum_{\pi \in\lrcorner_{n}} \prod_{i=1}^{n} C_{i \pi(i)}$ of the $n \times n$ matrix $C$.

\section{Review of Boson Stochastic Calculus}

Let $\mathscr{H}$ denote the Boson Fock space over the Hilbert space $h=L^{2}\left(\mathbb{R}_{+}\right)$. $\mathscr{H}$ is characterised as a Hilbert space equipped with a total family of exponential vectors 
$\psi(f), f \in h$ satisfying

$$
\langle\psi(f), \psi(g)\rangle=\exp \langle f, g\rangle, \quad f, g \in h
$$

to within unitary equivalence exchanging the exponential vectors. The vacuum is $\psi_{0}=\psi(0) ;$ the $n$-particle subspace $\mathscr{H}_{n}$ of $\mathscr{H}$ is the closed span of the vectors

$$
\left.\frac{\partial^{n}}{\partial \varepsilon_{1} \ldots \partial \varepsilon_{n}} \psi\left(\sum_{j=1}^{n} \varepsilon_{i} f_{j}\right)\right|_{\varepsilon_{1}=\ldots=\varepsilon_{n}=0}, \quad f_{1}, \ldots, f_{n} \in h .
$$

We denote by $\mathscr{E}$ the dense span of the exponential vectors. The Boson creation and annihilation operators corresponding to $f \in h$, the second quantisation of the unitary operator $U$ on $h$, and the differential second quantisation of the self-adjoint operator $T$ on $h$ are, respectively, the mutually adjoint operators defined on $\mathscr{E}$ by

$$
b(f) \psi(g)=\langle f, g\rangle \psi(g), \quad b^{\dagger}(f) \psi(g)=\left.\frac{d}{d \varepsilon} \psi(g+\varepsilon f)\right|_{\varepsilon=0},
$$

the unitary operator whose action on $\mathscr{E}$ is

$$
\Gamma(U) \psi(g)=\psi(U g),
$$

and the essentially self-adjoint operator on $\mathscr{E}$,

$$
d \Gamma(T) \psi(g)=-\left.i \frac{d}{d \varepsilon} \psi(\exp (i \varepsilon T) g)\right|_{\varepsilon=0}
$$

The closures of these operators act in the usual way in the $\mathscr{H}_{n}$.

For each Borel subset $S \subseteq \mathbb{R}_{+}$we let $P_{S}$ denote the operator on $h$ of multiplication by the indicator function $\chi_{S}$ of $S$, by $h_{S}$ the range of the projector $P_{S}$ and by $f_{S}$ the image of $f \in h$ under $P_{S}$. Corresponding to the natural projective decomposition $h=h_{S} \oplus h_{S c}$, we write

$$
\mathscr{H}=\mathscr{H}_{S} \otimes \mathscr{H}_{S^{c}}, \quad \psi(f)=\psi\left(f_{S}\right) \otimes \psi\left(f_{S^{c}}\right), \quad \mathscr{E}=\mathscr{E}_{S} \otimes \mathscr{E}_{S^{c}},
$$

where $\otimes$ denotes the algebraic tensor product.

Let there be given, once and for all, an initial Hilbert space $\mathscr{H}^{0}$, and set

$$
\tilde{\mathscr{H}}=\mathscr{H}^{0} \otimes \mathscr{H}, \quad \tilde{\mathscr{H}}_{S}=\mathscr{H}^{0} \otimes \mathscr{H}_{S}, \quad \widetilde{\mathscr{E}}=\mathscr{H}^{0} \otimes \underline{\mathscr{E}}, \quad \widetilde{\mathscr{E}}_{S}=\mathscr{H}^{0} \unrhd \mathscr{E}_{\mathrm{S}} .
$$

Following [4] rather than [1] we define an adapted process as a family $E=\left(E(t): t \in \mathbb{R}_{+}\right)$of operators in $\widetilde{\mathscr{H}}$ such that

a) for each $t \in \mathbb{R}_{+}, E(t)$ is the algebraic ampliation to $\widetilde{\mathscr{E}}_{[0, t]} \otimes \mathscr{E}_{(t, \infty)}$ of an operator in $\widetilde{\mathscr{H}}_{[0, t]}$ with domain $\widetilde{\mathscr{E}}_{[0, t]}$;

b) there is a family $E^{\dagger}=\left(E^{\dagger}(t): t \in \mathbb{R}_{+}\right)$also satisfying a) such that each $E^{\dagger}(t)$ is adjoint to $E(t)$.

The Boson annihilation and creation processes and the gauge process are the mutually adjoint processes $B, B^{\dagger}$ given by

$$
\left.B^{\#}(t)=I \underline{\otimes} b^{\#}\left(\chi_{[0}{ }^{\prime} t\right]\right)
$$

and the symmetric process $\Lambda$ given by

$$
\Lambda(t)=I \underline{\otimes} d \Gamma\left(P_{[0, t]}\right)
$$


respectively. As in [4] we may define the stochastic integral

$$
M(t)=\int_{0}^{t}\left(E_{1} d \Lambda+E_{2} d B^{\dagger}+E_{3} d B+E_{4} d s\right),
$$

first of simple (piecewise constant) processes, in such a way that

$$
M^{\dagger}(t)=\int_{0}^{t}\left(E_{1}^{\dagger} d \Lambda+E_{3}^{\dagger} d B^{\dagger}+E_{2}^{\dagger} d B+E_{4}^{\dagger} d s\right),
$$

and, for arbitrary $u, v \in \mathscr{H}^{0}, f, g \in h$,

$$
\langle u \otimes \psi(f), M(t) v \otimes \psi(g)\rangle=\int_{0}^{t}\left\langle u \otimes \psi(f),\left(\bar{f} g E_{1}+\bar{f} E_{2}+g E_{3}+E_{4}\right) v \otimes \psi(g)\right\rangle
$$

while, if also

$$
\begin{gathered}
M^{\prime}(t)=\int_{0}^{t}\left(E_{1}^{\prime} d \Lambda+E_{2}^{\prime} d B^{\dagger}+E_{3}^{\prime} d B+E_{4}^{\prime} d s\right), \\
\left\langle M(t) u \otimes \psi(f), M^{\prime}(t) v \otimes \psi(g)\right\rangle \\
=\int_{0}^{t}\left\{\left\langle M u \otimes \psi(f),\left(\bar{f} g E_{1}^{\prime}+\bar{f} E_{2}^{\prime}+g E_{3}^{\prime}+E_{4}^{\prime}\right) v \otimes \psi(g)\right\rangle\right. \\
+\left\langle\left(f \bar{g} E_{1}+\bar{g} E_{2}+f E_{3}+E_{4}\right) u \otimes \psi(f), M^{\prime} v \otimes \psi(g)\right\rangle \\
+\left\langle f E_{1} u \otimes \psi(f), g E_{1}^{\prime} v \otimes \psi(g)\right\rangle+\left\langle E_{1} u \otimes \psi(f), \bar{f} E_{2}^{\prime} v \otimes \psi(g)\right\rangle \\
\left.+\left\langle\bar{g} E_{2} u \otimes \psi(f), E_{1}^{\prime} v \otimes \psi(g)\right\rangle+\left\langle E_{2} u \otimes \psi(f), E_{2}^{\prime} v \otimes \psi(g)\right\rangle\right\} .
\end{gathered}
$$

Setting $M^{\prime}=M, g=f, v=u$ in (2.7), differentiating and making several uses of the inequality $2 \operatorname{Re}\left\langle\phi_{1}, \phi_{2}\right\rangle \leqq\left\|\phi_{1}\right\|^{2}+\left\|\phi_{2}\right\|^{2}$, we obtain

$$
\begin{aligned}
\frac{d}{d t}\|M(t) u \otimes \psi(f)\|^{2} \leqq & \left(1+3|f|^{2}\right)\|M u \otimes \psi(f)\|^{2} \\
& +3\left\|f E_{1} u \otimes \psi(f)\right\|^{2}+3\left\|E_{2} u \otimes \psi(f)\right\|^{2} \\
& +\left\|E_{3} u \otimes \psi(f)\right\|^{2}+\left\|E_{4} u \otimes \psi(f)\right\|^{2}
\end{aligned}
$$

Using the integrating factor $\exp \left\{-t-3 \int_{0}^{t}|f|^{2}\right\}$ this gives

$$
\begin{aligned}
\|M(t) u \otimes \psi(f)\|^{2} \leqq & \int_{0}^{t} \exp \left\{t-s+3 \int_{s}^{t}|f|^{2}\right\}\left[3\left\|f(s) E_{1}(s) u \otimes \psi(f)\right\|^{2}\right. \\
& +3\left\|E_{2}(s) u \otimes \psi(f)\right\|^{2}+\left\|E_{3}(s) u \otimes \psi(f)\right\|^{2} \\
& \left.+\left\|E_{4}(s) u \otimes \psi(f)\right\|^{2}\right] d s .
\end{aligned}
$$


From this inequality it is clear, by arguments similar to those of [4], that stochastic integration extends naturally to processes $E_{1}, E_{2}, E_{3}, E_{4}$ which, as well as being weakly measurable, satisfy the local square integrability conditions

$$
\int_{0}^{t}|f(s)|^{2}\left\|E_{1}(s) u \otimes \psi(f)\right\|^{2} d s<\infty, \quad \int_{0}^{t}\left\|E_{j}(s) u \otimes \psi(f)\right\|^{2}<\infty, \quad j=2,3,4
$$

for arbitrary $t \in \mathbb{R}_{+}, u \in \mathscr{H}^{0}, f \in h$, in such a way that (2.5), ..,(2.9) remain valid for the extended integral.

For stochastic differentials

$$
d M^{j}=E_{1}^{j} d \Lambda+E_{2}^{j} d B^{\dagger}+E_{3}^{j} d B+E_{4}^{j} d t, \quad j=1,2
$$

for which the coefficients $E_{k}^{j}$ and the $M^{j}$ are bounded operator valued processes satisfying, for all $t>0$,

$$
\sup _{0 \leqq s \leqq t} \max \left\{\left\|M^{j}(s)\right\|,\left\|E_{k}^{j}(s)\right\|, j=1,2, k=1,2,3,4\right\}<\infty,
$$

we obtain from (2.6) and (2.7) the Ito product formula

$$
d\left(M_{1} M_{2}\right)=d M_{1} \cdot M_{2}+M_{1} \cdot d M_{2}+d M_{1} \cdot d M_{2},
$$

where the right-hand side of (2.11) is evaluated by the rule that the basic differentials $d \Lambda, d A^{\dagger}, d A$, and $d t$ commute with adapted processes, and the correction term $d M_{1} \cdot d M_{2}$ is evaluated by combining this with extension by bilinearity of the multiplication table

\begin{tabular}{l|cccc} 
& $d \Lambda$ & $d B^{\dagger}$ & $d B$ & $d t$ \\
\hline$d \Lambda$ & $d \Lambda$ & $d B^{\dagger}$ & 0 & 0 \\
$d B^{\dagger}$ & 0 & 0 & 0 & 0 \\
$d B$ & $d B$ & $d t$ & 0 & 0 \\
$d t$ & 0 & 0 & 0 & 0
\end{tabular}

\section{Fermion Fields in Boson Fock Space}

In this section we construct Fermion field operators as Boson stochastic integrals using the Ito formula (2.11). For simplicity we take the initial space to be $\mathbb{C}$ so that $\tilde{\mathscr{H}}=\mathscr{H}$.

We define the reflection process $J=J^{\dagger}=\left(J(t): t \in \mathbb{R}_{+}\right)$by

$$
J(t)=\Gamma\left(-P_{[0, t]}+P_{(t, \infty)}\right), \quad t \in \mathbb{R}_{+} .
$$

Clearly $J$ is a self-adjoint, unitary adapted process, and $J^{2} \equiv I$.

Lemma 3.1. $J$ satisfies
a)

$$
J(s) J(t)=J(t) J(s) \quad\left(s, t \in \mathbb{R}_{+}\right),
$$$$
J(t) \psi_{0}=\psi_{0} \quad\left(t \in \mathbb{R}_{+}\right),
$$$$
\text { c) }
$$$$
d J=-2 J d \Lambda, \quad J(0)=I \text {. }
$$ 
Proof. a) and b) are immediate consequences of the definition. For c) we note first that since it is unitary, $J$ satisfies the first of conditions (2.10) so that $2 \int_{0}^{t} J d \Lambda$ is well defined. From (2.6), for arbitrary $t \in \mathbb{R}_{+}, f, g \in h$,

$$
\begin{aligned}
& \left\langle\psi(f),\left(I-2 \int_{0}^{t} J d \Lambda\right) \psi(g)\right\rangle=\langle\psi(f), \psi(g)\rangle-2 \int_{0}^{t} \bar{f} g\langle\psi(f), J \psi(g)\rangle \\
& =\langle\psi(f), \psi(g)\rangle-2 \int_{0}^{t} \bar{f}(s) g(s)\left\langle\psi(f), \psi\left(-g_{[0, s]}+g_{(s, \infty)}\right)\right\rangle d s \\
& =\langle\psi(f), \psi(g)\rangle+\int_{0}^{t} \frac{d}{d s} \exp \left(-\int_{0}^{s} \bar{f} g+\int_{s}^{\infty} \bar{f} g\right) d s \\
& =\exp \left(-\int_{0}^{t} \bar{f} g+\int_{t}^{\infty} \bar{f} g\right) \\
& =\langle\psi(f), J(t) \psi(g)\rangle .
\end{aligned}
$$

Let $\phi \in L_{\mathrm{loc}}^{2}\left(\mathbb{R}_{+}\right)$. We define the Fermion annihilation and creation processes of strength $\phi$ by

$$
F_{\phi}(t)=\int_{0}^{t} \overline{\phi(s)} J(s) d B(s), \quad F_{\phi}^{\dagger}(t)=\int_{0}^{t} \phi(s) J(s) d B^{\dagger}(s) ;
$$

since $J$ is a self-adjoint unitary operator $\phi J$ satisfies the local square integrability condition (2.10) and these are well defined mutually adjoint processes.

Lemma 3.2. For arbitrary $t \in \mathbb{R}_{+}, \phi \in L_{\text {loc }}^{2}\left(\mathbb{R}_{+}\right)$,

$$
J(t) F_{\phi}(t)+F_{\phi}(t) J(t)=0 .
$$

Proof. Since $\mathscr{E}$ is invariant under $J(t)$ both terms in (3.6) are operators on $\mathscr{E}$. For arbitrary $f, g \in h$, from (2.6),

$$
\begin{aligned}
\left\langle\psi(f),\left(J(t) F_{\phi}(t)+F_{\phi}(t) J(t)\right) \psi(g)\right\rangle=\left\langle J(t) \psi(f), F_{\phi}(t) \psi(g)\right\rangle \\
\quad+\left\langle\psi(f), F_{\phi}(t) J(t) \psi(g)\right\rangle \\
=\left\langle\psi\left(-f_{[0, t]}+f_{(t, \infty)}\right), F_{\phi}(t) \psi(g)\right\rangle+\left\langle\psi(f), F_{\phi}(t) \psi\left(-g_{[0, t]}+g_{(t, \infty)}\right)\right\rangle \\
=\int_{0}^{t}\left\{\left\langle\psi\left(-f_{[0, t]}+f_{(t, \infty)}\right), \bar{\phi}(s) g(s) J(s) \psi(g)\right\rangle\right. \\
\left.\quad-\left\langle\psi(f), \bar{\phi}(s) g(s) J(s) \psi\left(-g_{[0, t]}+g_{(t, \infty)}\right)\right\rangle\right\} d s \\
=\int_{0}^{t} \bar{\phi}(s) g(s)\{\langle J(t) \psi(f), J(s) \psi(g)\rangle-\langle J(s) \psi(f), J(t) \psi(g)\rangle\} d s=0,
\end{aligned}
$$

by (3.2). The second equation of (3.6) is proved similarly.

Theorem 3.3. The operators $F_{\phi}(t)$ are bounded and satisfy the canonical anticommutation relations

$$
\begin{gathered}
\left\{F_{\phi_{1}}(t), F_{\phi_{2}}(t)\right\}=0, \\
\left\{F_{\phi_{1}}(t), F_{\phi_{2}}^{\dagger}(t)\right\}=\int_{0}^{t} \bar{\phi}_{1} \phi_{2} .
\end{gathered}
$$


Proof. From (3.5) and the differentiated form of (2.7), for $f, g \in h$ and $\phi \in L_{\text {loc }}^{2}\left(\mathbb{R}_{+}\right)$,

$$
\begin{aligned}
\frac{d}{d t}\{ & \left.\left\langle F_{\phi}^{\dagger}(t) \psi(f), F_{\phi}^{\dagger}(t) \psi(g)\right\rangle+\left\langle F_{\phi}(t) \psi(f), F_{\phi}(t) \psi(g)\right\rangle\right\} \\
= & \left\langle\phi(t) \overline{g(t)} J(t) \psi(f), F_{\phi}^{\dagger}(t) \psi(g)\right\rangle+\left\langle F_{\phi}^{\dagger}(t) \psi(f), \phi(t) \overline{f(t)} J(t) \psi(g)\right\rangle \\
& +\left\langle\bar{\phi}(t) f(t) J(t) \psi(f), F_{\phi}(t) \psi(g)\right\rangle+\left\langle F_{\phi}(t) \psi(f), \bar{\phi}(t) g(t) J(t) \psi(g)\right\rangle \\
& +\langle\overline{\phi(t)} J(t) \psi(f), \overline{\phi(t)} J(t) \psi(g)\rangle \\
= & |\phi(t)|^{2}\langle\psi(f), \psi(g)\rangle,
\end{aligned}
$$

using (3.6). Thus, since $F(0)=0$,

$$
\left\langle F_{\phi}^{\dagger}(t) \psi(f), F_{\phi}^{\dagger}(t) \psi(g)\right\rangle+\left\langle F_{\phi}(t) \psi(f), F_{\phi}(t) \psi(g)\right\rangle=\int_{0}^{t}|\phi|^{2}\langle\psi(f), \psi(g)\rangle .
$$

From this it is clear that the operators $F_{\phi}^{\#}(t)$ are bounded and satisfy

and

$$
\left\|F_{\phi}^{\#}(t)\right\|^{2} \leqq \int_{0}^{t}|\phi|^{2}
$$

$$
\left\{F_{\phi}(t), F_{\phi}^{\dagger}(t)\right\}=\int_{0}^{t}|\phi|^{2} .
$$

Polarising we obtain (3.8). Now we can use Ito's formula to write

$$
d\left(F_{\phi}^{2}\right)=\bar{\phi}\left(J F_{\phi}+F_{\phi} J\right) d B=0,
$$

by Lemma 3.2. Since $F_{\phi}(0)=0$ we get $F_{\phi}^{2} \equiv 0$. Polarising gives (3.7).

\section{Identification of Boson and Fermion Fock Space}

We denote by $F, F^{\dagger}$ the processes $F_{\phi}, F_{\phi}^{\dagger}$ when $\phi \equiv 1$. Then

$$
d F^{\#}=J d B^{\#}, \quad d B^{\#}=J d F^{\#} .
$$

Stochastic integrals against $d F^{\#}$ are converted into integrals against $d B^{\#}$ using (4.1). For $\phi \in L_{\mathrm{loc}}^{2}\left(\mathbb{R}_{+}\right)$and $t \in \mathbb{R}_{+}$let

$$
B_{\phi}^{\#}(t)=\int_{0}^{t} \phi d B^{\#}(t)=b^{\#}\left(\phi_{[0, t]}\right) .
$$

Products of the operators $F_{\phi}(t)$ are meaningful since these are bounded operators, as are products of the $B_{\phi}(t)$ since $\mathscr{E}$ is invariant under these. We define products of the $B_{\phi}^{\dagger}(t)$ by adjunction.

Let $t \in \mathbb{R}_{+}$. We denote by $\Delta(n, t)$ the set

$$
\Delta(n, t)=\left\{\left(s_{1}, \ldots, s_{n}\right) \in[0, t]^{n}: 0 \leqq s_{1}<s_{2}<\ldots<s_{n} \leqq t\right\} .
$$

Lemma 4.1. Let $\phi_{1}, \ldots, \phi_{n} \in L_{\text {loc }}^{2}\left(\mathbb{R}_{+}\right)$. Then

$$
\begin{aligned}
& F_{\phi_{1}}^{\dagger}(t) \ldots F_{\phi_{n}}^{\dagger}(t) \psi_{0}=\int_{\Delta(n, t)} \operatorname{det}\left(\phi_{i}\left(s_{j}\right)\right) d B^{\dagger}\left(s_{1}\right) \ldots d B^{\dagger}\left(s_{n}\right) \psi_{0}, \\
& B_{\phi_{1}}^{\dagger}(t) \ldots B_{\phi_{n}}^{\dagger}(t) \psi_{0}=\int_{\Delta(n, t)} \operatorname{per}\left(\phi_{i}\left(s_{j}\right)\right) d F^{\dagger}\left(s_{1}\right) \ldots d F^{\dagger}\left(s_{n}\right) \psi_{0} .
\end{aligned}
$$


Proof. We prove (4.3) by induction on $n$, the case $n=1$ being a consequence of (4.1) and (3.3). Generally, by Ito's formula and Lemma 3.2, we have

$$
d\left(F_{\phi_{1}}^{\dagger} \ldots F_{\phi_{n+1}}^{\dagger}\right)=\sum_{j=1}^{n+1}(-1)^{n-j} \phi_{j} F_{\phi_{1}}^{\dagger} \ldots \hat{F}_{\phi_{j}}^{\dagger} \ldots F_{\phi_{n+1}}^{\dagger} J d B^{\dagger},
$$

and hence, making the inductive assumption that (4.3) holds, for $f \in h$,

$$
\begin{aligned}
& \left\langle\psi(f), F_{\phi_{1}}^{\dagger}(t) \ldots F_{\phi_{n+1}}^{\dagger}(t) \psi_{0}\right\rangle \\
& =\left\langle\psi(f), \int_{0}^{t} \sum_{j=1}^{n+1}(-1)^{n-j} \phi_{j}\left(s_{n+1}\right) F_{\phi_{1}}^{\dagger} \ldots \hat{F}_{\phi_{j}}^{\dagger} \ldots F_{\phi_{n+1}}^{\dagger}\left(s_{n+1}\right) d B^{\dagger}\left(s_{n+1}\right) \psi_{0}\right\rangle \\
& =\int_{0}^{t}\left\langle\psi(f), \overline{f\left(s_{n+1}\right)} \sum_{j=1}^{n+1}(-1)^{n-j} \phi_{j}\left(s_{n+1}\right) F_{\phi_{1}}^{\dagger} \ldots \hat{F}_{\phi_{j}}^{\dagger} \ldots F_{\phi_{n+1}}^{\dagger}\left(s_{n+1}\right) \psi_{0}\right\rangle d s_{n+1} \\
& =\left\langle\psi(f), \int_{\Delta(n+1, t)} \operatorname{det}\left[\phi_{i}\left(s_{j}\right)\right]_{i, j=1, \ldots, n+1} d B^{\dagger}\left(s_{1}\right) \ldots d B^{\dagger}\left(s_{n+1}\right) \psi_{0}\right\rangle,
\end{aligned}
$$

as is seen by expanding the determinant by its last column. Thus (4.3) holds for all $n$. As for (4.4), for $f \in h$ we have, by (4.2) and (2.2)

$$
\left\langle B_{\phi_{n}}(t) \ldots B_{\phi_{1}}(t) \psi(f), \psi_{0}\right\rangle=\prod_{j=1}^{n} \int_{0}^{t} \bar{f}_{\phi_{j}},
$$

while on the other hand, using (4.3) and (3.3) repeatedly

$$
\begin{aligned}
& \left\langle\psi(f), \int_{\Delta(n, t)} \operatorname{per}\left(\phi_{i}\left(s_{j}\right)\right) d F^{\dagger}\left(s_{1}\right) \ldots d F^{\dagger}\left(s_{n}\right) \psi_{0}\right\rangle \\
& =\left\langle\psi(f), \int_{0}^{t} \int_{\Delta\left(n-1, s_{n}\right)} \sum_{\pi \in\lrcorner_{n}} \prod_{j=1}^{n} \phi_{\pi(j)}\left(s_{j}\right) d F^{\dagger}\left(s_{1}\right) \ldots d F^{\dagger}\left(s_{n-1}\right) J d B^{\dagger}\left(s_{n}\right) \psi_{0}\right\rangle \\
& =\int_{0}^{t}\left\langle\psi(f), \bar{f}\left(s_{n}\right) \phi_{\pi(n)}\left(s_{n}\right) \int_{\Delta\left(n-1, s_{n}\right)} \sum_{\pi \in \Delta_{n}} \sum_{j=1}^{n-1} \phi_{\pi(j)}\left(s_{j}\right) d F^{\dagger}\left(s_{1}\right) \ldots d F^{\dagger}\left(s_{n-1}\right) \psi_{0}\right\rangle \\
& =\sum_{\pi \in \circlearrowleft_{n}} \int_{\Delta(n, t)} \prod_{j=1}^{n} \bar{f}\left(s_{j}\right) \phi_{\pi(j)}\left(s_{j}\right) \\
& =\prod_{j=1}^{n} \int_{0}^{t} \bar{f} \phi_{j} .
\end{aligned}
$$

Comparing (4.5) and (4.6) we see that $\psi_{0}$ is in the domain of the adjoint of $B_{\phi_{n}}(t) \ldots B_{\phi_{1}}(t)$, and that the action of the adjoint on $\psi_{0}$ is given by (4.4).

Since it is cyclic for the $B_{\phi}^{\dagger}(t)$, we deduce from (4.4) that the vacuum $\psi_{0}$ is cyclic for the operators $F^{\dagger}(t), t \in \mathbb{R}_{+}$, and hence for the $F_{\phi}^{\dagger}(t), \phi \in L_{\text {loc }}^{2}\left(\mathbb{R}_{+}\right), t \in \mathbb{R}_{+}$. Moreover the operators $F_{\phi}(t)$ annihilate the vacuum. Now the Fock representation $\left\{a^{\#}(f), f \in h\right\}$ of the canonical commutation relations in Fermion Fock space is characterised up to unitary equivalence by the existence of a cyclic vacuum vector annihilated by the annihilation operators $a(f)$. Furthermore the Fermion second quantisation $\Gamma_{F}(U)$ of a unitary operator $U$ on $h$ is the unique unitary operator which leaves the vacuum invariant and satisfies

$$
\Gamma_{F}(U) a^{\#}(f) \Gamma_{F}(U)^{-1}=a^{\#}(U f), \quad f \in h ;
$$


the differential second quantisation is then defined by

$$
d \Gamma_{F}(T)=-\left.i \frac{d}{d \varepsilon} \Gamma_{F}\left(e^{i \varepsilon T}\right)\right|_{\varepsilon=0} .
$$

We denote by $A$ and $A^{\dagger}$ the canonical Fermion annihilation and creation processes in Fermion Fock space constructed in [1]. Then we have

Theorem 4.3. There exists a unique unitary transformation $\Xi$ from $\mathscr{H}$ to Fermion Fock space over $L^{2}\left(\mathbb{R}_{+}\right)$such that

a) $\Xi \psi_{0}$ is the Fermion Fock vacuum vector

b) $\Xi F^{\#}(t) \Xi^{-1}=A^{\#}(t) \quad\left(t \in \mathbb{R}_{+}\right)$.

Furthermore, for each $t \in \mathbb{R}_{+}$,

$$
\Xi \Lambda(t) \Xi^{-1}=d \Gamma_{F}\left(P_{[0, t]}\right) .
$$

Proof. In view of the above, only (4.7) needs proof. An easy computation shows that, for arbitrary $f, g \in L^{2}\left(\mathbb{R}_{+}\right), \phi \in L_{\text {loc }}^{2}\left(\mathbb{R}_{+}\right), t \in \mathbb{R}_{+}$and real $\theta$,

$$
\begin{aligned}
& \left\langle\psi(f), \Gamma\left(e^{-i \theta} P_{[0, t]}+P_{(t, \infty)}\right) F_{\phi}(t) \Gamma\left(e^{i \theta} P_{[0, t]}+P_{(t, \infty)}\right) \psi(g)\right\rangle \\
& \quad=\left\langle\psi(f), F_{e^{i \theta} \phi}(t) \psi(g)\right\rangle,
\end{aligned}
$$

and hence that

$$
\Xi \Gamma\left(e^{i \theta} P_{[0, t]}+P_{(t, \infty)}\right) \Xi^{-1}=\Gamma_{F}\left(e^{i \theta} P_{[0, t]}+P_{(t, \infty)}\right) .
$$

Differentiating with respect to $\theta$ at $\theta=0$ we obtain (4.7).

Thus we may identify Fermion and Boson Fock space. From (2.12) and the fact that $J^{2}=I$ we immediately construct the corresponding Fermion Ito product formula, generalising that of [1] by the inclusion of the gauge process,

\begin{tabular}{l|cccc} 
& $d \Lambda$ & $d F^{\dagger}$ & $d F$ & $d t$ \\
\hline$d \Lambda$ & $d \Lambda$ & $d F^{\dagger}$ & 0 & 0 \\
$d F^{\dagger}$ & 0 & 0 & 0 & 0 \\
$d F$ & $d F$ & $d t$ & 0 & 0 \\
$d t$ & 0 & 0 & 0 & 0
\end{tabular}

In the implementation of (4.8) the differentials $d F$ and $d F^{\dagger}$ commute with even and anticommute with odd adapted processes where the parity is that of the $\mathbb{Z}_{2}$-graded structure of the even and odd $n$-particles subspace of $\mathscr{H}$. It is clear from Lemmas 4.1 and 4.2 that this grading transforms into that used in [1] under the transformation of Theorem 4.3.

\section{Solution of a Stochastic Differential Equation}

We consider the stochastic differential equation

$$
d U=U\left(L_{1} d \Lambda+L_{2} d F^{\dagger}+L_{3} d F+L_{4} d t\right), \quad U(0)=0,
$$

where the $L_{j}$ are ampliations of bounded operators (with which we identify them) on the initial space. Using (4.1) we write this as a Boson equation,

$$
d U=U\left(L_{1} d \Lambda+L_{2} J d B^{\dagger}+L_{3} J d B+L_{4} d t\right), \quad U(0)=0 .
$$


Necessary conditions for the existence of a unitary solution are obtained as in [4] by differentiating according to Ito's formula the relations $U^{\dagger} U=I, U U^{\dagger}=I$; they are

$$
\begin{gathered}
L_{1}+L_{1}^{\dagger}+L_{1}^{\dagger} L_{1}=0, \quad L_{1}+L_{1}^{\dagger}+L_{1} L_{1}^{\dagger}=0, \\
L_{3} J+J L_{2}^{\dagger}+J L_{2}^{\dagger} L_{1}=0,
\end{gathered}
$$

and (using the fact that $J$ commutes with $L_{2}$ )

$$
L_{4}+L_{4}^{\dagger}+L_{2}^{\dagger} L_{2}=0 \text {. }
$$

From (5.3) we obtain that

$$
L_{1}=W-I
$$

where $W$ is unitary. Setting $L_{2}=L,(5.4)$ implies that $L_{3} J=-J L^{\dagger} W=-L^{\dagger} W J$. From (5.5) we obtain $L_{4}=i H-\frac{1}{2} L^{\dagger} L$, where $H$ is self-adjoint. Thus (5.2) becomes

$$
d U=U\left((W-I) d \Lambda+L J d B^{\dagger}-L^{\dagger} W J d B+\left(i H-\frac{1}{2} L^{\dagger} L\right) d t\right), \quad U(0)=I .
$$

Theorem 5.1. Let $W, L, H \in B\left(\mathscr{H}^{0}\right)$ with $W$ unitary and $H$ self-adjoint. Then Eq. (5.6) has a unique solution.

Proof. The argument of [3] needs modification because $J$ in (5.6) comprises operators which are not in the initial space. Thus we define adapted processes $U_{n}$ inductively by $U_{0}(t) \equiv I$,

$$
U_{n}(t)=I+\int_{0}^{t} U_{n-1}(s)\left\{(W-I) d \Lambda+L J d B^{\dagger}-L^{\dagger} W J d B+\left(i H-\frac{1}{2} L^{\dagger} L\right) d s\right\} .
$$

Clearly $U_{0}(W-I)$ and $U_{0} L J, U_{0}\left(-L^{\dagger} W J\right), U_{0}\left(i H-\frac{1}{2} L^{\dagger} L\right)$ satisfy the local square integrability conditions (2.10). Assuming that $U_{n-1}(W-I)$ and $U_{n-1} L J$, $U_{n-1}\left(-L^{\dagger} W J\right), U_{n-1}\left(i H-\frac{1}{2} L^{\dagger} L\right)$ do likewise so that $U_{n}$ is defined, it follows that the stochastic integral $U_{n}-I$, hence $U_{n}$ also, is continuous in the sense [3] that, for arbitrary $u \in \mathscr{H}^{0}, f \in h, t \mapsto U_{n}(t) u \otimes \psi(f)$ is continuous, hence locally bounded. From this it follows that $U_{n}(W-I)$ and $U_{n} L J, U_{n}(-L W J), U_{n}\left(i H-\frac{1}{2} L L\right)$ satisfy (2.10) and $U_{n}$ is well defined for all $n$. We have

$$
\begin{aligned}
U_{n}(t)-U_{n-1}(t)= & \int_{0}^{t}\left(U_{n-1}(s)-U_{n-2}(s)\right) \\
& \cdot\left\{(W-I) d \Lambda+L J d B^{\dagger}-L^{\dagger} W J d B+\left(i H-\frac{1}{2} L^{\dagger} L\right) d s\right\}
\end{aligned}
$$

and

$$
\begin{aligned}
U_{n}^{\dagger}(t)-U_{n-1}^{\dagger}(t)= & \int_{0}^{t}\left\{\left(W^{\dagger}-I\right)\left(U_{n-1}(s)-U_{n-2}(s)\right) d \Lambda\right. \\
& -W^{\dagger} L J\left(U_{n-1}(s)-U_{n-2}(s)\right) d B^{\dagger}+L^{\dagger} J\left(U_{n-1}(s)-U_{n-2}(s)\right) d B \\
& \left.-\left(i H+\frac{1}{2} L^{\dagger} L\right)\left(U_{n-1}(s)-U_{n-2}(s)\right) d s\right\}
\end{aligned}
$$


We apply the estimate (2.9) to (5.8) to obtain

$$
\begin{aligned}
& \left\|\left(U_{n}(t)-U_{n-1}(t)\right) u \otimes \psi(f)\right\|^{2} \leqq \int_{0}^{t} \exp \left\{t-s+3 \int_{s}^{t}|f|^{2}\right\} \\
& \quad \cdot\left\{3|f(s)|^{2}\left\|\left(U_{n-1}(s)-U_{n-2}(s)\right)(W-I) u \otimes \psi(f)\right\|^{2}\right. \\
& \quad+3\left\|\left(U_{n-1}(s)-U_{n-2}(s)\right) L J(s) u \otimes \psi(f)\right\|^{2} \\
& \quad+\left\|\left(U_{n-1}(s)-U_{n-2}(s)\right) L^{\dagger} W J(s) u \otimes \psi(f)\right\|^{2} \\
& \quad+\|\left(U_{n-1}(s)-U_{n-2}(s)\left(i H-\frac{1}{2} L^{\dagger} L\right) u \otimes \psi(f) \|^{2}\right\} d s \\
& \leqq \\
& \quad 3 \int_{0}^{t} \exp \left\{t-s+3 \int_{s}^{t}|f|^{2}\right\}\left\{|f(s)|^{2}+1\right\} \\
& \quad \cdot\left\{\left\|\left(U_{n-1}(s)-U_{n-2}(s)\right)(W-I) u \otimes \psi(f)\right\|^{2}\right. \\
& \quad+\left\|\left(U_{n-1}(s)-U_{n-2}(s)\right) L u \otimes \psi(-f)\right\|^{2} \\
& \quad+\left\|\left(U_{n-1}(s)-U_{n-2}(s)\right) L^{\dagger} W U \otimes \psi(-f)\right\|^{2} \\
& \left.\quad+\left\|\left(U_{n-1}(s)-U_{n-2}(s)\right)\left(i H-\frac{1}{2} L^{\dagger} L\right) u \otimes \psi(f)\right\|^{2}\right\} d s,
\end{aligned}
$$

where we use adaptedness of $U_{n-1}-U_{n-2}$ to conclude that

$$
\begin{aligned}
& \left\|\left(U_{n-1}(s)-U_{n-2}(s)\right) L J(s) u \otimes \psi(f)\right\|^{2} \\
& \quad=\left\|\left(U_{n-1}(s)-U_{n-2}(s)\right) L u \otimes \psi\left(-f_{[0, s]}+f_{(s, \infty)}\right)\right\|^{2} \\
& \quad=\left\|\left(U_{n-1}(s)-U_{n-2}(s)\right) L u \otimes \psi(-f)\right\|^{2},
\end{aligned}
$$

and similarly

$$
\begin{aligned}
& \left\|\left(U_{n-1}(s)-U_{n-2}(s)\right) L^{\dagger} W J(s) u \otimes \psi(f)\right\|^{2} \\
& \quad=\left\|\left(U_{n-1}(s)-U_{n-2}(s)\right) L^{\dagger} W u \otimes \psi(-f)\right\|^{2} .
\end{aligned}
$$

We now iterate (5.10) obtaining

$$
\begin{aligned}
& \left\|\left(U_{n}(t)-U_{n-1}(t)\right) u \otimes \psi(f)\right\|^{2} \leqq(12 M)^{n} \int_{\Delta(n, t)} \exp \left\{t-s_{1}+3 \int_{s_{1}}^{t}|f|^{2}\right\} \\
& \cdot \prod_{j=1}^{n}\left\{\left|f\left(s_{j}\right)\right|^{2}+1\right\} d s_{1} \ldots d s_{n}\|u\|^{2}\|\psi(f)\|^{2},
\end{aligned}
$$

where $M=\max \left\{\|W-I\|^{2},\|L\|^{2},\left\|L^{\dagger} W\right\|^{2},\left\|i H-\frac{1}{2} L^{\dagger} L\right\|^{2}\right\}$. Since, as is easily seen by induction on $n$,

$$
\int_{\Delta(n, t)} \prod_{j=1}^{n}\left\{\left|f\left(s_{j}\right)\right|^{2}+1\right\} d s_{1} \ldots d s_{n}=\frac{1}{n !}\left(t+\left\|f_{[0, t]}\right\|^{2}\right),
$$

we see from (5.11) that, for $0 \leqq t \leqq T$,

$$
\left\|\left(U_{n}(t)-U_{n-1}(t)\right) u \otimes \psi(f)\right\|^{2} \leqq C(f, T) \frac{k(f, T)^{n}}{n !}\|u\|^{2},
$$

where $C(f, T), k(f, T)$ are positive constants. Thus $\sum_{n=0}^{\infty}\left(U_{n}(t)-U_{n-1}(t)\right) u \otimes \psi(f)$ converges, uniformly for $t \in[0, T]$; denote its limit by $U(t) u \otimes \psi(f)$. From (5.9) and (2.9) we see similarly that $\sum_{n=0}^{\infty}\left(U_{n}^{\dagger}(t)-U_{n-1}^{\dagger}(t)\right) u \otimes \psi(f)$ converges uniformly for 
$t \in[0, T]$, to $U^{\dagger}(t) u \otimes \psi(f)$ say. By continuity of the inner product $U$ and $U^{\dagger}$ are mutually adjoint processes. By uniformity of the convergence we may pass to the limit on both sides of (5.7) to obtain that (5.6) holds.

To prove uniqueness let $V$ be the difference of two solutions, then from the adjoint equation of (5.6) we have

$$
V^{\dagger}(t)=\int_{0}^{t}\left\{\left(W^{\dagger}-I\right) V^{\dagger} d \Lambda-W^{\dagger} L J V^{\dagger} d B^{\dagger}+L^{\dagger} J V^{\dagger} d B-\left(i H+\frac{1}{2} L^{\dagger} L\right) V^{\dagger} d s\right\} .
$$

From (2.9) we obtain that, for arbitrary $u \in \mathscr{H}^{0}, f \in h$, and $t \in \mathbb{R}_{+}$

$$
\left\|V^{\dagger}(t) u \otimes \psi(f)\right\|^{2} \leqq 3 \int_{0}^{t} \exp \left\{t-s+3 \int_{s}^{t}|f|^{2}\right\}\left\{1+|f(s)|^{2}\right\} M\left\|V^{\dagger}(s) u \otimes \psi(\mp f)\right\|^{2} d s .
$$

Since $V$ is a stochastic integral, $s \rightarrow\left\|V^{\dagger}(s) u \otimes \psi(\mp f)\right\|$ is continuous hence bounded on $[0, t]$. Thus we may iterate $(5.14)$, using $(5.12)$, to obtain that

$$
\left\|V^{\dagger}(t) u \otimes \psi(f)\right\|^{2} \leqq C^{\prime}(f, T) \frac{k^{\prime}(f, T)^{n}}{n !}\|u\|^{2} \underset{n}{\rightarrow} 0 .
$$

Thus $V \equiv 0$ and we have uniqueness.

\section{Unitarity of the Solution}

Lemma 6.1. The solution $U$ of (5.6) is coisometric.

Proof. Since

$$
U^{\dagger}(t)-I=\int_{0}^{t}\left\{\left(W^{\dagger}-I\right) U^{\dagger} d \Lambda-W^{\dagger} L J U^{\dagger} d B^{\dagger}+L^{\dagger} J U^{\dagger} d B-\left(i H+\frac{1}{2} L^{\dagger} L\right) U^{\dagger} d s\right\},
$$

we have from (2.7) that, for $u, v \in \mathscr{H}^{0}, f, g \in h, t \in \mathbb{R}_{+}$,

$$
\begin{aligned}
\left\langle\left(U^{\dagger}(t)-I\right) u \otimes \psi(f),\left(U^{\dagger}(t)-I\right) v \otimes \psi(g)\right\rangle \\
=-\int_{0}^{t}\left\{\left\langleu \otimes \psi(f),\left(\bar{f} g\left(W^{\dagger}-I\right) U^{\dagger}-\bar{f} W^{\dagger} L J U^{\dagger}\right.\right.\right. \\
\left.\left.\quad+g L^{\dagger} J U^{\dagger}-\left(i H+\frac{1}{2} L^{\dagger} L\right) U^{\dagger}\right) v \otimes \psi(g)\right\rangle \\
\quad+\left\langle\left(\bar{g} f\left(W^{\dagger}-I\right) U^{\dagger}-\bar{g} W^{\dagger} L J U^{\dagger}+f L^{\dagger} J U^{\dagger}\right.\right. \\
\left.\left.\quad-\left(i H+\frac{1}{2} L^{\dagger} L\right) U^{\dagger}\right) u \otimes \psi(f), v \otimes \psi(g)\right\rangle \\
=-\left\langle u \otimes \psi(f), U^{\dagger}(t) v \otimes \psi(g)\right\rangle-\left\langle U^{\dagger}(t) u \otimes \psi(f), v \otimes \psi(g)\right\rangle
\end{aligned}
$$

in view of (2.6). Hence

$$
\left\langle U^{\dagger}(t) u \otimes \psi(f), U^{\dagger}(t) v \otimes \psi(g)\right\rangle=\langle u \otimes \psi(f), v \otimes \psi(g)\rangle,
$$

and so $U^{\dagger}(t)$ is isometric.

Since $U(t)$ is coisometric it is bounded, uniformly for $t$ in finite intervals, and so we can apply (2.11) to write the differential

$$
\begin{aligned}
d(U J)= & d U \cdot J+U \cdot d J+d U \cdot d J=U(-(W+I) J d \Lambda \\
& \left.+L d B^{\dagger}+L^{\dagger} W d B+\left(i H-\frac{1}{2} L^{\dagger} L\right) J d t\right) .
\end{aligned}
$$


Theorem 6.2. The solution $U$ of (5.6) is unitary.

Proof. Again we modify the corresponding arguments of $[3,4]$. By an extension of the Hellinger Toeplitz theorem [4] the operators $u \mapsto U(t) u \otimes \psi(f)$, $u \mapsto U^{\dagger}(t) u \otimes \psi(f)$ are bounded from $\mathscr{H}^{0}$ to $\tilde{\mathscr{H}}$ for each fixed $f \in h$ and $t \in \mathbb{R}_{+}$. By adaptedness of $U$ the same is true of $u \mapsto U(t) J(t) u \otimes \psi(f)$. Hence we can define bounded operators $K_{j}(f, g)(t)$ on $\mathscr{H}^{0}$ for $j=1,2,3,4, f, g \in h$ and $t \in \mathbb{R}_{+}$by

$$
\left.\begin{array}{l}
\left\langle u, K_{1}(f, g)(t) v\right\rangle=\langle U(t) u \otimes \psi(f), U(t) v \otimes \psi(g)\rangle \\
\left\langle u, K_{2}(f, g)(t) v\right\rangle=\langle U(t) J(t) u \otimes \psi(f), U(t) v \otimes \psi(g)\rangle \\
\left\langle u, K_{3}(f, g)(t) v\right\rangle=\langle U(t) u \otimes \psi(f), U(t) J(t) v \otimes \psi(g)\rangle \\
\left\langle u, K_{4}(f, g)(t) v\right\rangle=\langle U(t) J(t) u \otimes \psi(f), U(t) J(t) v \otimes \psi(g)\rangle
\end{array}\right\}
$$

From (2.7) and (2.5) we have that, for arbitrary $u, v \in \mathscr{H}^{0}$,

$$
\begin{aligned}
\frac{d}{d t}\langle u, & \left.K_{1}(f, g)(t) v\right\rangle \\
= & \left\langle u,\left[\left\{\bar{f} g\left(W^{\dagger}-I\right)-\left(i H+\frac{1}{2} L^{\dagger} L\right)\right\} K_{1}(f, g)+\bar{f} g\left(W^{\dagger}-I\right) K_{1}(f, g)(W-I)\right.\right. \\
& +K_{1}(f, g)\left\{\bar{f} g(W-I)+\left(i H-\frac{1}{2} L^{\dagger} L\right)\right\}+\left\{g L^{\dagger}-\bar{f} W^{\dagger} L\right\} K_{2}(f, g) \\
& +g L^{\dagger} K_{2}(f, g)(W-I)+K_{3}(f, g)\left\{\bar{f} L-g L^{\dagger} W\right\} \\
& \left.\left.+\bar{f}\left(W^{\dagger}-I\right) K_{3}(f, g) L+L^{\dagger} K_{4}(f, g) L\right] v\right\rangle .
\end{aligned}
$$

Similarly, using (6.1), we find that

$$
\begin{aligned}
& \frac{d}{d t}\left\langle u, K_{2}(f, g) v\right\rangle=\left\langle u,\left[-\left\{\bar{f} g\left(W^{\dagger}+I\right)+\left(i H+\frac{1}{2} L^{\dagger} L\right)\right\} K_{2}(f, g)\right.\right. \\
&+K_{2}(f, g)\left\{\bar{f} g(W-I)+\left(i H-\frac{1}{2} L^{\dagger} L\right)\right\}-\bar{f} g\left(W^{\dagger}+I\right) K_{2}(f, g)(W-I) \\
&+\left\{\bar{f} W^{\dagger} L+g L^{\dagger}\right\} K_{1}(f, g)+g L^{\dagger} K_{1}(f, g)(W-I) \\
&\left.\left.+L^{\dagger} K_{3}(f, g) L+K_{4}(f, g)\left\{\bar{f} L-g L^{\dagger} W\right\}-\bar{f}\left(W^{\dagger}+I\right) K_{4}(f, g) L\right] v\right\rangle, \quad(6.4) \\
& \frac{d}{d t}\left\langle u, K_{3}(f, g) v\right\rangle \\
&=\left\langle u,\left[\left\{\bar{f} g\left(W^{\dagger}-I\right)-\left(i H+\frac{1}{2} L^{\dagger} L\right)\right\} K_{3}(f, g)+K_{3}(f, g)\{-\bar{f} g(W+I)\right.\right. \\
&\left.+\left(i H-\frac{1}{2} L^{\dagger} L\right)\right\} \\
&-\bar{f} g\left(W^{\dagger}-I\right) K_{3}(f, g)(W+I)+K_{1}(f, g)\left\{\bar{f} L+g L^{\dagger} W\right\}+\bar{f}\left(W^{\dagger}-I\right) K_{1}(f, g) L \\
&\left.\left.+L^{\dagger} K_{2}(f, g) L+\left\{g L^{\dagger}-\bar{f} W^{\dagger} L\right\} K_{4}(f, g)-g L^{\dagger} K_{4}(f, g)(W+I)\right] v\right\rangle, \\
& \quad \frac{d}{d t}\left\langle u, K_{4}(f, g) v\right\rangle=\left\langle u,\left[-\left\{\bar{f} g\left(W^{\dagger}+I\right)+i H+\frac{1}{2} L^{\dagger} L\right\} K_{4}(f, g)\right.\right. \\
& \quad-K_{4}(f, g)\left\{\bar{f} g(W+I)-i H+\frac{1}{2} L^{\dagger} L\right\}+\bar{f} g\left(W^{\dagger}+I\right) K_{4}(f, g)(W+I) \\
& \quad+L^{\dagger} K_{1}(f, g) L+K_{2}(f, g)\left\{\bar{f} L+g L^{\dagger} W\right\}-\bar{f}\left(W^{\dagger}+I\right) K_{2}(f, g) L \\
&\left.\left.\quad+\left\{g L^{\dagger}+\bar{f} W^{\dagger} L\right\} K_{3}(f, g)-g L^{\dagger} K_{3}(f, g)(W+I)\right] v\right\rangle .
\end{aligned}
$$


We regard (6.3), (6.4), (6.5), and (6.6) as simultaneous ordinary differential equations for the four operator-valued functions $K_{j}(f, g), j=1,2,3,4$. A particular solution satisfying the initial condition

$$
K_{j}(f, g)(0)=\exp \langle f, g\rangle I, \quad j=1,2,3,4
$$

is given by

$$
\begin{aligned}
& K_{1}(f, g)(t)=K_{4}(f, g)(t) \equiv \exp \langle f, g\rangle I, \\
& K_{2}(f, g)(t)=K_{3}(f, g)(t)=\exp \left\{-2 \int_{0}^{t} \bar{f} g+\langle f, g\rangle\right\} I,
\end{aligned}
$$

as may be verified by direct substitution. If we restrict $f$ and $g$ to be locally bounded functions, then the solution with given initial condition is unique. In particular $K_{1}(f, g)$ is necessarily given by (6.8), and so

$$
\langle U(t) u \otimes \psi(f), U(t) v \otimes \psi(g)\rangle=\langle u, v\rangle \exp \langle f, g\rangle=\langle u \otimes \psi(f), v \otimes \psi(g)\rangle
$$

for all such $f$ and $g$, and $u, v \in \mathscr{H}^{0}$. Since $U(t)$ is bounded, (6.9) must hold without restriction on $f$ and $g$. Hence $U(t)$ is isometric. Combining this with Lemma 7.1 we conclude that $U(t)$ is unitary.

\section{References}

1. Applebaum, D.B., Hudson, R.L.: Fermion Ito's formula and stochastic evolutions. Commun. Math. Phys. 96, 473-496 (1984)

2. Barnett, C., Streater, R.F., Wilde, I.: The Ito-Clifford integral I. J. Funct. Anal. 48, 172-212 (1982)

3. Hudson, R.L., Parthasarathy, K.R.: Quantum Ito's formula and stochastic evolutions. Commun. Math. Phys. 93, 301-323 (1984)

4. Hudson, R.L., Parthasarathy, K.R.: Stochastic dilations of uniformly continuous completely positive semigroups. Acta Appl. Math. 2, 353-398 (1984)

5. Hudson, R.L., Parthasarathy, K.R.: Generalised Weyl operators. In: Stochastic analysis and applications. Truman, A., Williams, D. (eds.) Lecture Notes in Mathematics, Vol. 1095. Berlin, Heidelberg, New York: Springer 1984

6. Meyer, P.A.: Fock space and probability theory, preprint

7. Meyer, P.A.: Private communication

8. Applebaum, D.B.: Fermion Ito's formula II, preprint

9. Garbaczewski, P., Rzewuski, J.: On generating functionals for antisymmetric functions and their applications in quantum field theory. Rep. Math. Phys. 6, 431-444 (1974)

Garbaczewski, P.: Representations of the CAR generated by representations of the CCR Fock case. Commun. Math. Phys. 43, 131-136 (1976), Fermi states of Bose systems in three space dimensions. J. Math. Phys. 26, 490-494 (1985)

10. Garbaczewski, P.: Some aspects of the Boson-Fermion (in)equivalence: A remark on the paper by Hudson and Parthasarathy. Bibos preprint 68

Communicated by H. Araki

Received June 28, 1985; in revised form November 2, 1985 